\title{
Prof. Dr. sc. Günter Herrendörfer 65 Jahre
}

Am 7.11.2003 vollendet Prof. Dr. sc. Günter Herrendörfer sein 65. Lebensjahr. Dazu gratulieren ihm seine Freunde, viele Kollegen, Schüler und seine ehemaligen Mitarbeiter ganz herzlich. Seine Wurzeln hat er im ostpreußischen Schippenbeil, das er kriegsbedingt verlassen musste. Im Ostseebad Heiligendamm fand er ein neues Zuhause.

Nach Schulbesuch in Heiligendamm und in Bad Doberan (Abitur) sowie einem praktischen Jahr als Landarbeiter in der LPG Hohenfelde, studierte er an der Universität Leipzig Wirtschaftsmathematik. Das Studium schloss er 1964 erfolgreich ab. Eine Zäsur in seinem Leben stellte ein Unfall im Kindesalter dar, der zu einer lebenslangen Beeinträchtigung seines Sehvermögens führte.

Günter Herrendörfer's wissenschaftliche Laufbahn begann als Aspirant an der Akademie der Landwirtschaftswissenschaften der DDR im Tierzuchtinstitut in Dummerstorf. An diesem Ort hat er sein gesamtes wissenschaftliches Leben lang, bis zu seiner vorzeitigen Pensionierung im Jahre 1999, erfolgreich gewirkt. Als wissenschaftlicher Mitarbeiter arbeitete er zunächst in der Abteilung „Biomathematik und Versuchsplanung“. 1968 promovierte er an der Landwirtschaftsakademie in Berlin zum Dr. rer. nat. mit dem Thema: „Beiträge zur Versuchsplanung der Schätzung der Heritabilitätskoeffizienten von Merkmalen unserer Haustiere (Rind, Schwein, Schaf)“. Im Jahre 1978 erfolgte die Promotion B mit dem Thema „Versuchplanung im linearen Modell - Schätzung von Varianzkomponenten und eingeschränkte Messwerterfassung von Mittelwerten und Regressionskoeffizienten“ an der Martin-Luther-Universität Halle-Wittenberg.

Die Fakultas docendi erhielt er 1991 von der Universität Rostock für das Fach Biometrie und Versuchswesen.

Von 1986 bis 1990 leitete er als wissenschaftlicher Abteilungsleiter die Abteilung Populationsgenetik des Bereiches Züchtungsforschung im Forschungszentrum für Tierproduktion Dummerstorf. Nach der Evaluierung des Forschungszentrums durch den Wissenschaftsrat, im Zusammenhang mit der Wiedervereinigung, übernahm er die Leitung des Forschungsbereiches „Genetik und Biometie“ im neugegründeten Forschungsinstitut für die Biologie landwirtschaftlicher Nutztiere (FBN). In Anerkennung seiner herausragenden wissenschaftlichen Leistungen und seiner Einflussnahme auf die Verbesserung von Versuchsplanung und -auswertung in der Tierzuchtforschung wurde er 1990 zum Professor der Akademie der Landwirtschaftswissenschaften berufen. Wie bereits aus den Themen der Promotionen ersichtlich, hat Günter Herrendörfer in seinem wissenschaftlichen Leben immer an zwei Fronten gekämpft um die Brücke zwischen der Theorie und der praktischen Umsetzung zu schlagen. Seine Hauptarbeitsgebiete lassen sich in folgenden Punkten zusammenfassen: 
- Versuchsplanung

- Robustheit statistischer Verfahren

- Optimierung von Zuchtsystemen

- Indexselektion.

Aus diesen Arbeitsgebieten resultieren mehr als 250 wissenschaftliche Publikationen als Erstund Mitautor. Unter seiner Leitung sind 10 Dissertationen auf den Gebieten der mathematischen Statistik und der angewandten Züchtungsmethodik entstanden.

Besonders hervorzuheben sind 18 Monographien bzw. Buchtitel, die er herausgegeben oder an denen er maßgeblich mitgewirkt hat. Darunter sind die Monographie „Verfahrensbibliothek - Versuchsplanung und -auswertung“, die 1981 in der DDR und 1996 im OldenbourgVerlag, München, erschien, 1982 die „Statistische Versuchsplanung“ (Herrendörfer und Schüler) und 1990 die Monographie „Populationsgenetik und Züchtungsmethodik“ (Hrsg. Rasch und Herrendörfer). Nicht zu vergessen ist die Herausgabe der Dummerstorfer Schriftenreihe „Genetische Probleme in der Tierzucht“, von der 29 verschiedene Hefte vorliegen.

Günter Herrendörfer hat sein Wissen nicht nur in Publikationen niedergelegt, sondern auch eine umfangreiche Lehrtätigkeit an der Universität Rostock ausgeübt. Seit 1964 hielt er Vorlesungen sowohl vor Studenten der Landwirtschaft als auch vor Studenten der Mathematik in den Fächern Biometrie und Mathematische Statistik.

Die Stärken von Günter Herrendörfer liegen in der Symbiose von mathematischen Theorien, die er als Mathematiker weiterentwickelte, ihr Umsetzen in der Versuchsplanung und -auswertung sowie ihre Nutzung für die Züchtungsmethodik. Günter Herrendörfer hat sich stets dafür eingesetzt, dass auf allen Gebieten der Tierzucht und Genetik Versuche und Experimente den Anforderungen der angewandten Statistik entsprachen. So wurden z. B. im damaligen Dummerstorfer Forschungszentrum alle Forschungsvorhaben hinsichtlich der Planung und Auswertung von Versuchen durch die Abteilung Biometrie begutachtet. In der Weiterbildung von Doktoranden hat er sich viele Jahre persönlich engagiert und bis heute vertritt er die Meinung, dass eine unkritische Übernahme von Computerergebnissen, auch wenn sie mit der modernsten Software erarbeitet wurden, zu Fehlern führt, wenn die mathematisch-statistischen Kenntnisse des Anwenders unzureichend sind.

Seine sehr zahlreichen Gutachten zu Dissertationen und Forschungsberichten zeichneten sich immer durch konstruktive Kritik bei der Bewertung von Versuchsplanung und -auswertung aus und zeigten auf diesem Gebiet oft die Schwachstellen derartiger Arbeiten.

Die Umsetzung seiner Kenntnisse in die praktische Zuchtplanung und Populationsgenetik lassen sich durch Begriffe wie die Entwicklung der Methoden der simulierten Selektion, der Genotyp-Umwelt Wechselwirkungen, der Maternaleffekte, der Langzeitselektion und der Optimierung des Zuchtfortschrittes unter dem Aspekt ihrer Anwendung bei allen landwirtschaftlichen Nutztieren und der Pflanzenzucht, beschreiben.

Seine breiten wissenschaftlichen Arbeitsgebiete führten dazu, dass er seit 1983 im Redaktionskollegium des „Archiv für Tierzucht“ und seit 1990 in der Redaktion der „Biometrischen Zeitschrift“ tätig ist. Als Spezialist für die Begutachtung von Manuskripten mit viel „Mathematik“ steht er bis heute unserem „Archiv“ zur Verfügung.

Prof. Günter Herrendörfer beendete 1999 aus gesundheitlichen Gründen vorzeitig seine berufliche Tätigkeit. Seine Hobbys, die Gartenarbeit und das Angeln, füllen einen großen Teil seiner Freizeit aus. Das er diesen, seinen Hobbys, noch recht lange nachgehen kann, das wünschen ihm Freunde, Kollegen und Schüler. Sie alle danken Günter Herrendörfer für die Hilfe und Zusammenarbeit, die stets fördernd, offen und kritisch war und wünschen uns mit ihm noch viele persönliche Begegnungen und ihm Gesundheit und erholsame Stunden im Kreise seiner Familie. 